\title{
Angular momentum control in coordinated behaviors
}

\author{
Victor Zordan \\ University of California, Riverside
}

\begin{abstract}
This paper explores the many uses of angular momentum regulation and its role in the synthesis of coordinated motion generated with physically based characters. Previous investigations in biomechanics, robotics, and animation are discussed and a straightforward organization is described for distinguishing the needs and control approaches of various behaviors including stepping, walking and standing balance. Emphasis is placed on creating robust response to large disturbances as well as on the types of characteristic movements that can be generated through the control of angular momentum.
\end{abstract}

Keywords: Character animation; Physics models; Behavior control

\section{Introduction}

Natural movement of humans reveals a high degree of coordination in which the entire body moves in concert to perform a given task. Even seemingly simple behaviors such as basic walking and balancing show that the arms, trunk, and legs work together to produce the signature motions we identify as humanlike. Making characters (and robots) that move with the sophistication of humans has been a long standing goal of many researchers over several decades. And recent advances in computer animation, biomechanics, and robotics have begun to explore the ability to produce whole-body coordinated motions in the control of humanoids. Beyond ad-hoc heuristics that function to perform a given task, the drive to uncover the core principles of human coordination is an increasingly interesting motivation for research in the control of animation for characters.

One exciting trend in the investigation of human motion control is the exploration of the contribution of angular momentum in the production of behaviors. Clear examples in humans, such as windmilling (to aid in balance), point to the obvious presence of angular momentum in movement with gross rotational components. Less obvious is that precise regulation appears in human motions with seemingly little rotational component. For example, recent studies in biomechanics have shown that careful angular momentum regulation appears in normal walking [1]. This finding is particularly interesting because it is not obvious that walking should include very precise control over whole-body rotation. With such compelling evidence and findings in biomechanics, there is surprisingly little exploration of angular momentum as it applies to control in physics based human animation. 
In this paper, we revisit the effects of angular movement in various tasks, both observed in humans and controlled in humanoid robots and simulations. We summarize the related findings from biomechanics, robotics, and animation and draw correlations between them in order to propose a division of angular momentum controllers into two categories based on behavior. We highlight testbeds with example implementations for each category as well as describe a supervisor which uses angular momentum to perform planning. We conclude with a discussion of the open questions and possibilities related to the use of angular momentum in the control of coordinated behavior.

\section{Related Work}

\subsection{Angular Momentum Control in Biomechanics}

Popovic, Herr and several colleagues [1-5] have collectively performed the most thorough study of the role of angular momentum in human movement to date. They postulate that whole-body (spin) angular momentum may be regulated directly by the central nervous system. In their investigations, they have studied walking motion in depth and report observing surprisingly small angular momentum values in straightline walking which lead to very small (2 degree) angular excursions over entire cycles of normal subjects' walking. Based on such observations, they propose that walking is regulated to have Zero Spin (ZS) angular momentum about the center of mass (CM). For walking and other "ZS" behaviors, their hypothesis is that both angular momentum and its time derivative are regulated to remain close to zero. Several of their various findings support this hypothesis. Along with data analysis and models of human subjects, these researchers have also spelled out the value of regulating angular momentum in control for humanoid robots and they have implemented and described a handful of simulations.

Along with ZS behaviors such as walking, this research team has also suggested that there are behaviors which have Non-Zero Spin (NZS) angular momentum [1]. Within this group they include "large and rapid turning motions" as well as motions in response to "sufficiently large disturbances". Interestingly, they also report that, upon entering the conditions of these behaviors, human subjects exhibit an observable switch in control strategy away from the ZS control described for walking [5]. One of the few related investigations thus far is in the strategies used for turning in walking behaviors which indicates that a significant non-zero spin angular momentum is induced (largely by the swing leg) during normal turning. Also, in a recent paper [3], they propose that humans modulate spin momentum "to enhance CM control." Through simulation they show that a simplified, seven-link humanoid can induce momentum to bring its $\mathrm{CM}$ within its support after starting from a statically instable balance state (i.e. where the CM starts from rest outside of the support.) Their finding is supported by human examples of the same phenomena and indicates a distinct need to be able to induce angular momentum in order to regain stable balance. 
We postulate that humans may be regulating angular momentum constantly to carry out a wide class of rotationally rich "NZS" behaviors. Herr and Popovic support this hypothesis in their analysis of a human performing a "Hula" action - a dynamic but sustained and stable motion which shows an order of magnitude more angular momentum than walking [4]. Beyond this sparse set of examples, such NZS behaviors have not been studied (with respect to angular momentum and control) by the biomechanics research community to date.

\subsection{Angular Momentum Control in Robotics}

Robotics researchers have postulated ways in which control of angular momentum can increase controller robustness while lead to coordinated motions for humanoid robots. Much of the recent effort in this area follows from the work of Kajita et al. [6]. Their "resolved momentum control" strategy appears to be the first in which angular momentum is controlled simultaneously with linear momentum (i.e. control over the $\mathrm{CM}$ ), added deliberately to make behavior control easier. In this work, they tout the benefit of combined momenta to "describe the macroscopic behavior of the entire robot" independent of its structure. In this work, and similar follow-on work [7], they show results applied to humanoid robots where the angular momentum about the vertical axis is driven to near zero for activities such as a kick and walking.

Goswami and Kallem [8] support angular momentum guidance as a robust method for controlling biped robots. They suggest that a controller might directly guide the time derivative of angular momentum, $\dot{H}$, and state that this term is "physically central to rotational instability and intuitively more transparent to the phenomena of tipping and tumbling" than derived quantities such as ZMP and centroid moment pivot (defined below.) They also describe theoretical strategies for regaining stability, each reducing to a unified control approach: return to a condition where $\dot{H}=0$, that is leading from an NZS to ZS state.

Several papers appear in the robotics literature that employ some variant of angular momentum control. While the proposed control laws vary, all propose simple heuristic-based control laws crafted for specific effects. Kajita and colleagues set the angular momentum to be zero for control of their humanoid robot [6]. Abdallah and Goswami use a momentum controller to absorb disturbance effects [9]. They suggest that during large external perturbations humans absorb impact by preserving momentum for a specified period of time. After the impact has been absorbed, the character recovers its posture. Stephens employs a bang-bang control to use the body like a flywheel, applying maximum torque as necessary [10]. One common theme in all of these papers is that each treats the control of angular momentum as a damper (that is, to dissipate a disturbance). Collectively, they show that simple control laws can be very effective. Notably however, no exploration of sustained NZS behavior control appears in robotics, to our knowledge. 


\subsection{Angular Momentum Control in Animation}

Most of the full-body control work that has been proposed in computer animation to date has employed some mechanism for controlling the CM and/or its derivatives often by generating a reference position and, possibly, a reference velocity. This has lead to a host of controllers for activities including walking, leaping, running, and standing (balance). While heuristic, tuned controllers have been shown to be very robust, they often appear robotic. In recent years, such manual approaches have given way to more automatic controllers which compute joint torques for the full-body based on a relatively small set of control inputs - usually some combination of reference joint trajectories, CM trajectories, and constraints (e.g. to keep contact forces within friction limits). While it can be shown that controlling the $\mathrm{CM}$ while maintaining the ground reaction forces (GRF) to remain within a friction cone can yield indirect changes in angular momentum, this phenomena is generally discouraged in an optimization framework in lieu of energy efficiency unless it is ultimately "necessary". That is, only under extreme conditions (e.g. when the CM is close to or on the boundary of the support) will a character exhibit noticeable changes associated with angular momentum.

Only a handful of papers have investigated control which drives angular momentum in an explicit manner. Kudoh and colleagues propose a controller that employs angular momentum to constrain the (predicted) zero moment point (ZMP) to remain in the support [11]. Their animations exhibit a reaction similar to those seen in CM control with GRF constraints because their system induces momentum only when the ZMP is at the edge of the support. Macchietto et al. [12] uses a sustained NZS controller that produces response to disturbances that resist fast changes to the ZMP while directing it to a safe desired location. More detail on this research is outlined in Section 4. de Lasa et al. [13] follow ZS behavior characteristic described in biomechanics $(H=0)$ in their control for locomotion but also induce momentum about the vertical axis $\left(H_{z}>0\right)$ for turning jumps, consistent with NZS control. Ye and Liu [14] apply a ZS strategy for all behaviors and succeed in producing a large number of motions, all with visible coordinated rotation across the body. [15] employs a hybrid approach similar to Kajita et al. [6] for generating steps $\left(H_{z}=0\right)$. Using this technique, control over vertical spin momentum is shown to yield characteristic arm movement for walking without the need for special treatment or a specific joint reference trajectory.

\section{Mechanics of momentum}

In the absence of external force, the linear and angular momenta of a system, denoted $L$ and $H$, are conserved. Momentum change comes from the ground reaction forces (GRF) and force due to gravity as well as additional external forces if they are present. In the case of flat ground and no additional external forces, we can summarize the momenta change simply. Assuming the aggregate GRF force, $f$, is applied at position, $p$, then $\dot{L}=m g+f$ and $\dot{H}=s \times f$ where $g$ is 


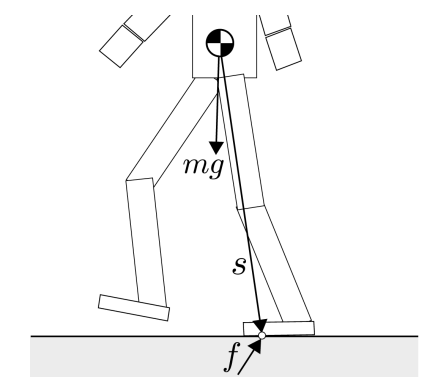

Fig. 1. Static force analysis for a standing character.

the gravitational constant, $m$ is the total mass, and $c$ is the CM of the character. $s=p-c$ as seen in Figure 1 .

By simple inspection, we can see that the derivative of the linear momentum is the same as the mass-scaled CM acceleration. Note this coupling implies that control over the CM (and its time derivatives) as has been seen in most previous control approaches for locomotion across disciplines is equivalent to control of linear momentum (and its derivatives), assuming mass is constant. We can also see that angular (spin) momentum change completely describes the relationship between the position of the center of mass, $c$, and the aggregate GRF, $f$, applied at point $p$. Finally, together, along with $f$, these two momenta rates can be integrated to yield the complete rigid motion of the character about its CM.

As a quick aside, what is the point $p$ ? In biomechanics, this point is commonly called the center of pressure $(\mathrm{CP})$ or the point where aggregate continuous forces is applied equivalently (i.e. without adding additional torque). In robotics, this point is often considered synonymous with the ZMP, and it is, in the case of flat horizontal ground. However, the ZMP can be shown to differ from the CP when the ground is irregular $[8,16]$. Formally, the ZMP is the point where the net moment about the GRF has zero-value in the horizontal components, which is the $\mathrm{CP}$ - on flat ground. In literature in robotics and animation, the ZMP is often "predicted" to be outside of the support - which is physically inconsistent with its definition. Because there is less confusion and misuse of the meaning of the term, we opt to use the term "CP" in lieu of "ZMP" whenever possible. See [16] for a lengthier discussion on the topic.

In the related biomechanics and robotics literature, researchers point out that for a given (non-zero) GRF only one possible position for $p$ will yield zero angular momentum change (assuming flat ground.) They call this point the centroid moment pivot (CMP) $[16,8,2]$. Unlike the ZMP or $\mathrm{CP}$, this point is a derived value which is defined in a manner in which it may or may not reside within the support. The beauty of this term is that, just as the location of the CM projected on the ground plane can indicate stability in a standing character, the CMP can be used to indicate rotational stability. If the CMP is within the support, then the CP could theoretically be aligned with the CMP and a zero angular momentum could be realized. However, if the CMP is computed to be outside of the support 
then the CP cannot meet the CMP. To avoid tipping, angular momentum must be added to the system and the character will invariably experience some amount of whole-body rotation (for non-zero GRF.)

This characteristic leads us to a mechanism for cleanly dividing the two classes of control identified, namely ZS behaviors and NZS behaviors. As the CMP crosses the edge of the support, we infer that the control changes from one to the other depending on the direction. We organize the remainder of this paper based on this distinction. First, we highlight an approach for controlling NZS behaviors through an analysis of response to large disturbances in regaining stability in balance. Next, we explore aspects of ZS behaviors, specifically as they apply in the production of steps and walking. Finally, we describe a mechanism developed for planning which exploits knowledge about the angular momentum independent of the behavior control approach used.

\section{Balance Control}

In our recent investigation in standing balance, we explore a method for controlling the $\mathrm{CP}$ and the $\mathrm{CM}$ simultaneously in order to respond in the presence of large disturbances [12]. To this extent, we propose a multi-objective technique which synthesizes animation according to three objectives, namely an objective for tracking a reference motion (taken from human motion capture) and two objectives that control changes in linear and angular momenta. By converting each objective into a set of desired accelerations, we combine them into a single quadratic optimization problem which can be solved in a straightforward and efficient manner. The resulting accelerations are applied to the character based on torques computed from a floating-base hybrid inverse dynamics calculation. In our results, we show a suite of animations which reveal full-body coordinated response to a host of different conditions and inputs.

One of the contributions of this work is in the method used to control the $\mathrm{CP}$ and $\mathrm{CM}$ simultaneously. Exploiting the described relationship between momenta and stability, at each run of the optimization we convert target $\mathrm{CP}$ and $\mathrm{CM}$ values to a pair of desired changes in momenta. By accounting for the dependencies created through the requirement of a unified GRF, the two desired momenta terms are computed to create a consistent desired change in the character's center of mass and full-body rotation. In contrast, computing these terms separately would lead to the objectives competing and both could not be fully realized. Notably, de Lasa and colleagues get around this issue in their work through a prioritization approach, placing the linear momentum objective ahead of the angular momentum objective in priority [13].

From the perspective of ZS/NZS angular momentum classification, our balance control approach falls succinctly in the NZS category because the technique is inherently asking for non-zero momenta changes which lead to non-zero angular momentum behavior. In contrast to other controllers, our approach is unique in that we purposefully drive angular momentum changes that will control the $\mathrm{CP}$ to move in a smooth, deliberate manner throughout the entire behavior. Ku- 
doh et al [11] instead put barrier constraints on the ZMP which is quite similar to constraining the CM with friction limits. As Kudoh's controller constrains the ZMP to stay within the support, it only "turns on" angular momentum control when the ZMP hits the support boundary. In this condition, the situation is already at an extreme, and bang-bang like control response is needed to quickly move the ZMP away from the support edge. However, human motion doesn't appear to support this approach [3]. Instead a smooth, less extreme ZMP trajectory seems desirable based on the data. A more common strategy for responding to disturbances is to damp the movement of the ZMP or the angular momentum present but without explicitly guiding the ZMP/CP it can still head to the edge and lead to rotational instability. These approaches treat the NZS case as if it is simply a transition back to ZS control.

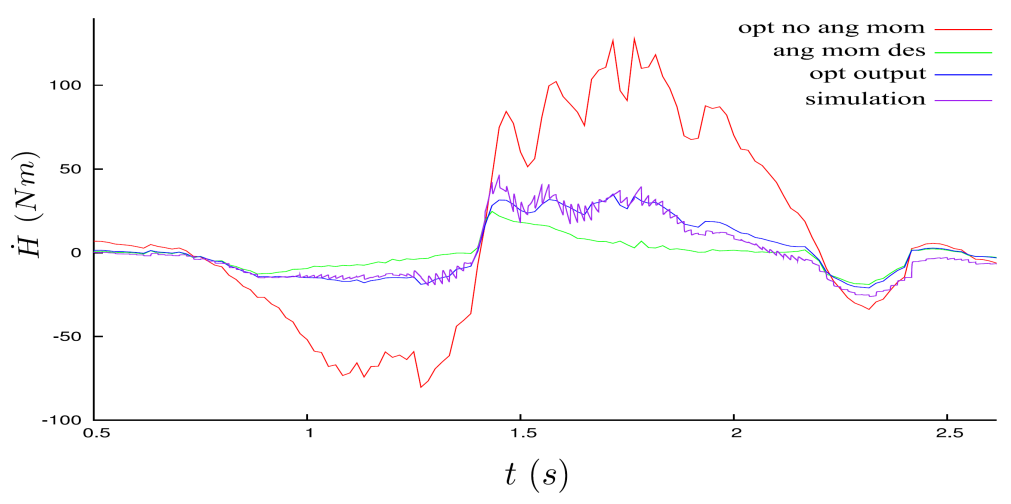

Fig. 2. Response to a large impulse. Angular momentum change observed under the no-control condition (red) is highly reduced when control is applied. The discrepancy between the desired (green) and the optimization solution (blue) accounts for the other objectives, tracking and moving the CM. The simulation (purple) closely follows the optimization request. The jagged simulation response is due to the fact that the optimization is updated at a lower frequency, revealing that assumptions about GRF and so on quickly become stale - this could be remedied if we were willing to run the optimization in lockstep with the simulation.

In our control approach, we bring the (ZMP/)CP toward the center of support, but do so gradually and smoothly. By damping its motion, we avoid abrupt changes that lead quickly to rotational instability (e.g. tipping) and by persistently guiding the $\mathrm{CP}$ back to the center of support the controller directs the motion of the character to a safer stance. When we turn off the angular momentum objective, our system exhibits balance which is both less robust based on the size of impact and contains fewer of the rotational artifacts we observe in humans related to balance, for example raising the arms in response to a push backward. The extent of our system's ability to control the angular momentum is highlighted in Figure 2. 


\section{Locomotion}

In a more recent publication [15], we explore a momentum-based strategy that is aligned with the ZS category of control. In that paper, we expand the described framework for balance control to produce stepping behaviors produced by specifying trajectories for the $\mathrm{CM}$ and the swing foot. The desired motion of the swing foot is translated into a joint-angle reference trajectory and in doing so we can use the same solver implemented for the balance paper. However, instead of guiding the $\mathrm{CP}$ as we did previously, we specify angular momentum directly similar to the scheme described by Kajita et al. in their resolved momentum control paper [6]. Specifically, we enforce $\dot{H}_{z}=0$ while the other spin momentum terms are uncontrolled. The effect of this controller is to restrict twist (yaw) rotation, thereby maintaining a consistent facing direction while allowing rotation in roll and pitch. Since one goal was to stop in presence of disturbances, the latter is a means of allowing the character to yield to any momentum changes following an impact which is a behavior identified as humanlike by Abdallah and Goswami [9]

By specifying a series of equal length steps, this controller can also produce a walking gait. While the method of angular momentum regulation is more relaxed than other control techniques (such as [13] and others from robotics), the controller successfully produces walking behavior with distinct humanlike characteristics (see Figure 3). Further, the approach does not require parameters that are specific to the character and so we can use the same system to generate walking for characters with different morphologies (see Figure 4).

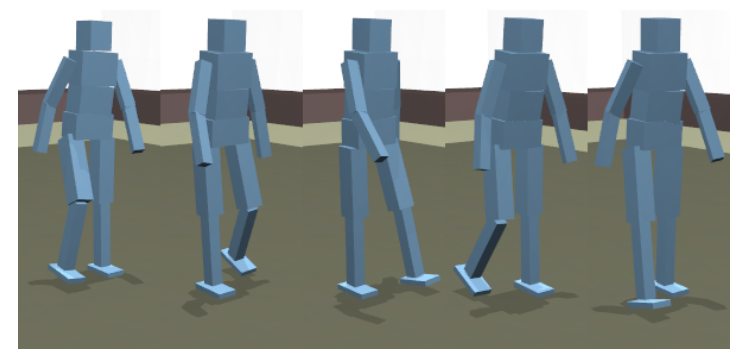

Fig. 3. Arm swing is produced which reasonably imitates what is present in humans even though the tracked trajectory for this animation is a single arms-at-side pose.

\section{Planning}

As a final example, we showcase a method for employing angular momentum at the planning stage of control. In our recent work in stepping, we developed a supervisor which guides the step behavior controller. By combining and expanding the momenta change equations outlined in Section 3, we can compute the 


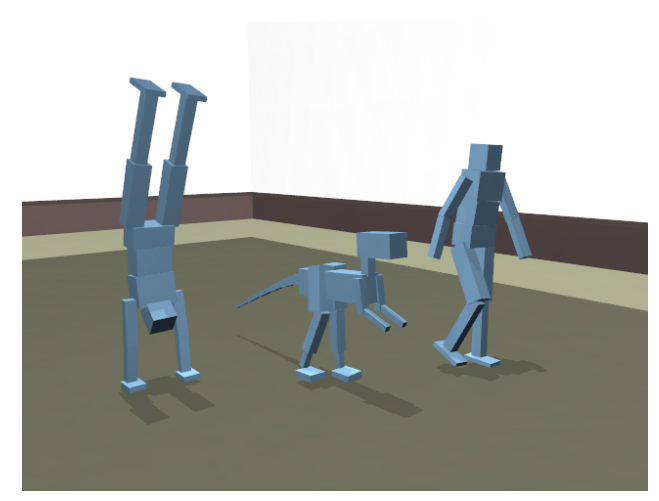

Fig. 4. Various walking gaits. In these animations, additional leg swing (left) and tail motion (center) act functionally the same as the arm swing movements seen in the basic walker (right). These "secondary" motions are generated automatically from angular momentum control. (Parameters for the dinosaur model from [17].)

position $p$ on the ground plane, assuming we're given the remaining terms. Using $p$, we can determine when the support is about to rotate and, therefore, when a step is necessary to prevent tipping. To provide anticipatory control, we add dampers which dissipate momenta, as such $\dot{L}_{d e s}=-d_{l} \cdot L$ and $\dot{H}_{d e s}=-d_{h} \cdot H$. The result is a momentum driven, easily tunable predictor for when and where to take a step. Namely, we compute $p_{\text {des }}$ as:

$$
\begin{gathered}
p_{x_{d e s}}=c_{x}+\frac{d_{l} \cdot L_{x}}{f_{z}} c_{z}+\frac{d_{h} \cdot H_{y}}{f_{z}} \\
p_{y_{d e s}}=c_{y}+\frac{d_{l} \cdot L_{y}}{f_{z}} c_{z}-\frac{d_{h} \cdot H_{x}}{f_{z}} .
\end{gathered}
$$

where $f_{z}=\dot{L}_{z}+m g$. If this value is outside of the support, we take a step. With a scaled set of gain values, we can also use these equations to choose where to step.

The strength and uniqueness of this supervisor is that it takes into account the angular momentum present which can be substantial following a large disturbance. Another perk of this supervisory controller is that it is easily tunable to create desired effects since it only depends on a small number of gain values [15]. In contrast, other step supervisors including inverted pendulum and capture point models $[18,19]$ ignore angular momentum in choosing where to step. As we show in the paper, if we simply drop the angular momentum term from the calculation, the supervisor reduces to a very similar structure as the capture point and we see a significant drop in the capability of the resulting character in handling disturbances [15]. We conclude from our findings that consideration of angular momentum is quite valuable in high level planning as well as low level behavior control. 


\section{Discussion}

In our momentum explorations thus far, we have shown how it is possible to use momentum to produce coordinated motion such as balance, stepping, and walking through a multi-objective framework. Several key observations were made during the course of these efforts. Among the most important, we found that balance and locomotion control is a problem concerned with the aggregate dynamics of the articulated-body system; what happens with each individual body within this black box is less important than the combined effect of all. In this way, control can be delegated to other objectives (for example, the style of the animation take from motion capture). Control inputs for momentum are inherently low dimensional independent of the dimensionality (or morphology) of the character which seems to pair nicely with this finding. Next, while rotational movement adds a great deal of visual finesse to a motion, we have seen that it is relatively easy to solicit with guidance over the angular momentum as evidenced by the many successful yet simple control laws proposed thus far. With such a strong potential for producing rich, realistic movement, it is our goal to thoroughly explore the maintenance of angular momentum as an explicit and deliberate feature of control.

The concepts described in this paper highlight the importance of angular momentum control over whole-body coordinated behaviors in all human activities. The underlying hypothesis is that control over angular momentum is a critical mechanism which is being employed in nature. This hypothesis is supported by compelling evidence published mostly within the last five years in biomechanics and robotics. To date, all but a small handful of animation researchers have ignored or greatly simplified the whole-body rotational aspects of behavior control. Recent findings in regards to control over angular momentum and its ability to create coordinated and more humanlike movement make it difficult to continue to justify arguments for this choice.

To the animation community, perhaps the greatest value of momentum-based is simplification: full body, coordinated control is reduced to the specification of two basic, well-behaved momenta signals. Practical techniques for controlling these signals have been proposed and successfully tested. And now, a new set of questions arise. How are humans using momentum control to produce movements, if indeed they are? How can animators best harness benefits of this phenomena? What are the driving signals and pertinent features? What are the limits of this control paradigm? Thus far, we have seen very little in terms of speculations but yet there is evidence of tremendous untapped potential, suggesting great opportunity.

\section{Conclusion}

In this paper, we highlight findings from the literature and our explorations with respect to the relationship of angular momentum and coordinated behavior in physics-based character animation control. We present a basic ZS/NZS classification for distinguishing behaviors that exhibit distinct strategies in angular 
momentum regulation and discuss recently proposed techniques for using angular momentum in control in the context of this breakdown as well as summarize our experimental results and those of others in animation. Notably, sustained NZS control is virtually non-existent across disciplines thus far.

We conclude that angular momentum control is a general tool for guiding coordinated action through the purposeful, deliberate changes to whole-body angular momentum. Biomechanists have shown that humans carefully regulate angular momentum in common activities and preliminary investigations from several disciplines support that angular momentum control, even in simple forms, can achieve a high degree of coordination and robustness in responses to a variety of conditions. Further, resulting secondary effects caused by such controllers, such as arm swing in humanoids, produce motions that bear strong likenesses to those observed in natural, real-world movement. We predict that with such mechanisms for control, physically based character motion can become more flexible and believable in addition to new behavior controllers becoming easier to construct.

Acknowledgments. The author wishes to acknowledge the co-authors of related research, C. C. (James) Wu, Adriano Macchietto, and Christian Shelton for their input and help with the production of figures. Also, thanks to Paul Kry and Ambarish Goswami for helpful discussions that lead to a more integrated understanding of this material.

\section{References}

1. Popovic, M., Hofmann, A., Herr, H.: Angular momentum regulation during human walking: biomechanics and control. In: Proceedings of the IEEE International Conference on Robotics and Automation, Citeseer (2004) 2405-2411

2. Popovic, M., Hofmann, A., Herr, H.: Zero spin angular momentum control: definition and applicability. In: 2004 4th IEEE/RAS International Conference on Humanoid Robots. (2004) 478-493

3. Hofmann, A., Popovic, M., Herr, H.: Exploiting angular momentum to enhance bipedal center-of-mass control. IEEE Trans. Rob. Autom (2007)

4. Herr, H., Popovic, M.: Angular momentum in human walking. Journal of Experimental Biology 211 (2008)

5. Farrell, M., Herr, H.: Angular Momentum Primitives for Human Turning: Control Implications for Biped Robots. IEEE Humanoids Conference (2008)

6. Kajita, S., Kanehiro, F., Kaneko, K., Fujiwara, K., Harada, K., Yokoi, K., Hirukawa, H.: Resolved momentum control: humanoid motion planning based on the linear and angular momentum. Intelligent Robots and Systems (IROS) (2003)

7. Ahn, K., Oh, Y.: Walking Control of a Humanoid Robot via Explicit and Stable CoM Manipulation with the Angular Momentum Resolution. In: 2006 IEEE/RSJ International Conference on Intelligent Robots and Systems. (2006) 2478-2483

8. Goswami, A., Kallem, V.: Rate of change of angular momentum and balance maintenance of biped robots. IEEE Int. Conf. Robotics and Automation (ICRA) (2004) 
9. Abdallah, M., Goswami, A.: A biomechanically motivated two-phase strategy for biped upright balance control. In: IEEE Int. Conf. Robotics and Automation. (2005)

10. Stephens, B.: Humanoid Push Recovery. In: IEEE-RAS International Conference on Humanoid Robots. (2007)

11. Kudoh, S., Komura, T., Ikeuchi, K.: Stepping motion for a humanlike character to maintain balance against large perturbations. In: IEEE Int. Conf. Robotics and Automation. (2006)

12. Macchietto, A., Zordan, V., Shelton, C.R.: Momentum control for balance. ACM Transactions on Graphics 28 (2009) 80:1-80:8

13. de Lasa, M., Mordatch, I., Hertzmann, A.: Feature-based locomotion controllers. ACM Transactions on Graphics 29 (2010) 131:1-131:10

14. Ye, Y., Liu, C.K.: Optimal feedback control for character animation using an abstract model. ACM Transactions on Graphics 29 (2010) 74:1-74:9

15. Wu, C.C., Zordan, V.: Goal-directed stepping with momentum control. In: ACM SIGGRAPH / Eurographics Symposium on Computer Animation. (2010) 113-118

16. Popovic, M., Goswami, A., Herr, H.: Ground Reference Points in Legged Locomotion: Definitions, Biological Trajectories and Control Implications. The International Journal of Robotics Research 24 (2005)

17. Coros, S., Beaudoin, P., van de Panne, M.: Robust task-based control policies for physics-based characters. ACM Transactions on Graphics 28 (2009)

18. Pratt, J., Carff, J., Drakunov, S., Goswami, A.: Capture Point: A Step toward Humanoid Push Recovery. Proceedings of the IEEE-RAS/RSJ International Conference on Humanoid Robots (2006)

19. Rebula, J., Canas, F., Pratt, J., Goswami, A.: Learning Capture Points for Bipedal Push Recovery. Robotics and Automation, 2008. ICRA 2008. IEEE International Conference on (2008) 1774-1774 